\title{
Pancreatic transplantation: surgical technique, normal radiological appearances and complications
}

\author{
Frances A. Hampson - Susan J. Freeman • \\ Julia Ertner • Martin Drage • Andrew Butler • \\ Chris J. Watson • Ashley S. Shaw
}

Received: 12 May 2010 /Revised: 5 August 2010 /Accepted: 18 September 2010 /Published online: 30 October 2010

(C) European Society of Radiology 2010

\begin{abstract}
Pancreas transplantation is a surgical treatment for diabetes mellitus. More than 23,000 pancreas transplants have now been reported to the International Transplant Registry (IPTR). Early diagnosis and therapy for graft-related complications are essential for graft survival. Radiologists must therefore understand the surgical procedure and the potential complications. During the course of this review, we will illustrate the normal postoperative anatomy and the imaging appearances of common potential complications.
\end{abstract}

Keywords Simultaneous pancreas kidney transplantation . Imaging

\section{Introduction}

Pancreas transplantation is a surgical treatment for diabetes mellitus (DM). Most recipients have type $1 \mathrm{DM}$ but $7.7 \%$ have type 2 DM [1, 2]. Transplant obviates the need for insulin treatment and is associated with an improvement in quality of life and life expectancy. The euglycaemia obtained has been shown to prevent and sometimes

F. A. Hampson $(\bowtie) \cdot$ S. J. Freeman · A. S. Shaw

Department of Radiology, Addenbrooke's Hospital,

Hills Road,

Cambridge CB2 0QQ, UK

e-mail: frances.hampson@addenbrookes.nhs.uk

J. Ertner $\cdot$ A. Butler $\cdot$ C. J. Watson

Department of Surgery, Addenbrooke's Hospital,

Cambridge, UK

M. Drage

Department of Transplantation, Guy's and St Thomas' NHS Trust, London, UK improve the secondary complications of DM including nephropathy, neuropathy, retinopathy and macrovascular disease.

The first whole-pancreas transplant was performed in the University of Minnesota, USA in 1966 [3] and, with improvements in surgical technique and immunosuppressive therapy, increasing numbers of successful transplants are performed. More than 23,000 pancreas transplants have now been reported to the International Transplant Registry (IPTR) [1]. In the UK the number of pancreas transplants has steadily increased from 30 , in 1998-1999, to 209 , in 2007-2008.

Following pancreas transplantation, it is essential that any graft-related complications are diagnosed early to ensure prompt treatment and optimal survival of both graft and patient. In order to successfully interpret the imaging, it is essential that the surgical procedure, normal postoperative appearances and potential complications are understood by the reporting radiologists. The clinically suspected complication should direct the imaging modality to be employed. In particular, the most appropriate CT protocols should be utilised to ensure that the maximum information is obtained at the lowest possible radiation dose. Within this review we will illustrate the normal postoperative anatomy and the imaging appearances of the commonest potential complications.

\section{Types of pancreas transplant}

Pancreas transplantation is most commonly performed in conjunction with a kidney transplant, a procedure referred to as simultaneous pancreas-kidney (SPK) transplantation. SPK transplants are considered in patients with insulindependent DM and chronic renal failure either requiring or 
imminently requiring dialysis. SPK recipients have improved 10-year survival when compared with diabetic patients receiving kidney transplantation alone: mean 23.4 years versus 12.9 years, respectively [4].

Of 209 pancreas transplants performed in the UK in 2007-2008, 173 (83\%) were SPK transplants donated following brain death (DBD), although an increasing number of pancreases in the UK are donated following cardiac death (DCD). Alternatively, solitary pancreas transplants may be performed [5], usually in patients who have already had a kidney transplant often from a living donor: so-called pancreas after kidney transplants (PAK). Approximately $10 \%$ of cases involve PAKs. Occasionally, both organs (distal pancreas segment and whole kidney) may come from a living donor [6]. Only $0.5 \%$ pancreatic transplants are from living donors [1].

Less than $10 \%$ involve pancreas transplantation alone (PTA). Thirty percent of those given a PTA will eventually need a renal transplant because of the adverse cumulative effects of immunosuppression with calcineurin inhibitors [7]. PTA is performed for patients with labile, poorly controlled insulin-dependent type $1 \mathrm{DM}$ with stable renal function. The principal indication for a PTA is lifethreatening hypoglycaemic unawareness. The long-term advantages of good glycaemic control must be weighed against the risks of major surgery and the complications of long-term immunosuppressive therapy.

\section{Surgical technique}

At our institution, the pancreas transplant is placed intraperitoneally, lying on the right side of the pelvis; the kidney is placed in the left iliac fossa and is extra peritoneal to facilitate biopsy (Fig. 1).

The donor pancreas is retrieved en bloc with the duodenum, which is transected and stapled proximally just beyond the pylorus and distally in the third part of the duodenum. The common bile duct is also divided and ligated at the level of the duodenum. At the recipient hospital the donor iliac artery bifurcation is anastomosed to the stumps of the splenic and superior mesenteric arteries of the pancreas bloc; this facilitates implantation, during which the donor common iliac artery is anastomosed to the recipient artery, usually the common iliac in our centre; thus the donor iliac artery forms an inverted Y-graft conduit.

Venous outflow is via the donor portal vein, which is anastomosed end-to-side to the recipient inferior vena cava; in some centres the pancreas is implanted to the external iliac vessels, in which case the portal vein may need to be extended by anastomosing donor iliac vein to act as a conduit. However, the use of venous extension grafts

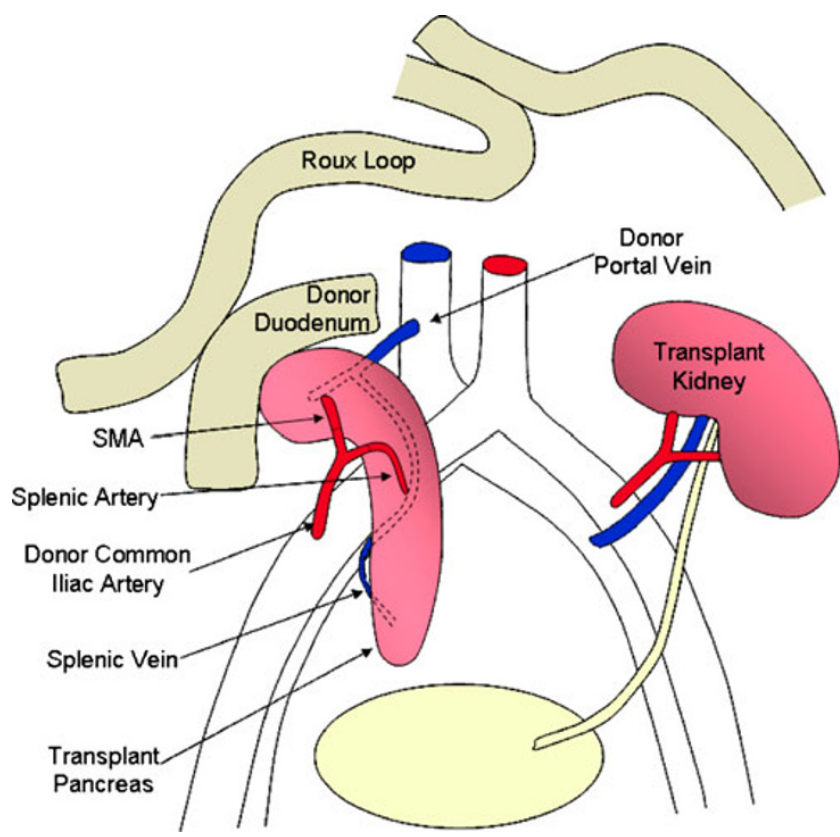

Fig. 1 Schematic diagram of SPK transplant, with enteric drainage

increases the risk of graft thrombosis. A modification to this technique is portal rather than systemic venous drainage of the endocrine pancreas by anastomosing the portal vein to the superior mesenteric vein.

Exocrine secretions drain via the donor duodenum, which is nowadays commonly anastomosed to recipient small bowel, either directly or, as in our centre, to a Roux loop of recipient jejunum via a side-to-side anastomosis. Alternatively, the donor duodenum may be anastomosed directly to the bladder [8]. In all modifications the recipient's native pancreas is left untouched.

When both organs come from a living donor a distal pancreatectomy is performed. The pancreas is divided at the neck. Distally the main trunks of the splenic artery and vein are divided proximal to the splenic branches. Proximally the splenic artery is divided at its origin off the coeliac trunk and the splenic vein is divided at the confluence with the superior mesenteric vein. The donor splenic artery is then anastomosed end to side to a recipient iliac artery and the donor splenic vein is anastomosed end to side to a recipient iliac vein. Exocrine secretions drain via an enteric route or the bladder $[6,9,10]$.

\section{Imaging techniques and normal anatomy}

Ultrasound (US), computed tomography (CT) and magnetic resonance imaging (MRI) are all used to assess the pancreas following transplantation. Conventional angiography is largely reserved to confirm vascular complications and permits endovascular therapy. 
US is of limited value in the initial assessment of the pancreatic parenchyma, as the transplant margins are often poorly defined. In addition, views may be limited by overlying bowel gas due to the intra-peritoneal position of the transplant pancreas. Assuming good views are obtained, Doppler US may be of great use in evaluating the patency and calibre of the arterial and venous grafts (Fig. 2). The resistive index has been shown to be an unreliable indicator of rejection and therefore biopsy is the diagnostic "gold standard" [11, 12]. US-guided biopsies have a high success rate [13], ensuring the modality's continued application in this field. There is, however, an increased risk of bleeding associated with pancreas biopsy compared with transplant kidney biopsy as the pancreas is intraperitoneal, thus bleeding will not be tamponaded by either the peritoneum or by the organ capsule. Pancreatic leaks are the other complication which deters clinicians, and for these reasons biopsy of the renal transplant may be used as a surrogate marker for pancreatic rejection [14], although asynchronous rejection may occur.

Complete and reliable evaluation of the pancreatic parenchyma and the vascular and enteric anastomoses is best achieved with cross-sectional imaging. Both CT and MRI have their individual merits in this situation; $\mathrm{CT}$ is often more readily available and permits images to be acquired much more quickly than MRI. This is particularly of value in unstable patients.

CT images should be acquired in three phases: unenhanced, arterial and venous. The unenhanced images are used to locate the pancreas transplant and to identify vascular thromboses and haematomas, due to their increased attenuation. The arterial-phase study should be performed in an early angiographic phase, focused on the pancreas transplant. In our institution this is achieved by bolus tracking over the abdominal aorta and imaging at $7 \mathrm{~s}$ after the arrival of contrast medium. The arterial phase

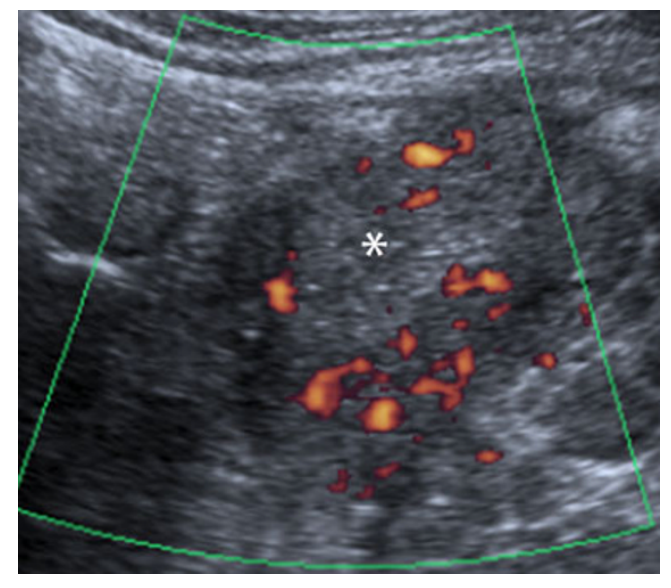

Fig. 2 US image revealing normal homogeneous echotexture of the pancreatic graft (white asterisk) in the right iliac fossa, with normal vascularity on power Doppler often demonstrates parenchymal enhancement and early venous drainage, depending on the transit time across the graft pancreas. The venous phase obtained $50 \mathrm{~s}$ postinjection (Fig. 3) of the whole abdomen and pelvis demonstrates graft venous drainage, parenchymal enhancement and fluid collections. Multi-detector CT (MDCT) enables 3D reconstructions (Fig. 4) of the vascular structures, which may enhance the detection of vascular complications.

SPK transplants may result in asymmetrical enhancement of the common iliac veins. This appearance is known as pseudothrombosis of the iliac vein [15]. It results from the rapid transit time across the transplant kidney and early opacification of the iliac vein in comparison with the contralateral side.

Dynamic contrast enhanced MRI allows superior evaluation of the pancreatic parenchyma. Graft perfusion may be assessed and areas of necrosis demonstrated. Evaluation of the enteric anastomoses may be more difficult. MR angiography is considered to be inferior to CT angiography because of its limited spatial resolution.

MR cholangiopancreatography (MRCP) may be used to detect duct abnormalities. In cases of recurrent graft pancreatitis it can reveal both causes and consequences, e.g. pancreatic necrosis. Collections resulting from pancreatic leaks may be also demonstrated. Secretin-augmented MRCP has been used to show reduced exocrine function that may correlate with pancreatic rejection [16].

One factor that may limit the application of both CT and MRI is impaired renal transplant function, which is not uncommon in the immediate post-operative period. Under these circumstances both intravenous iodinated contrast media and gadolinium-based contrast media should be used with caution because of the respective risks of contrastinduced nephropathy and nephrogenic systemic fibrosis. Both CT and MRI may be applied without contrast media,

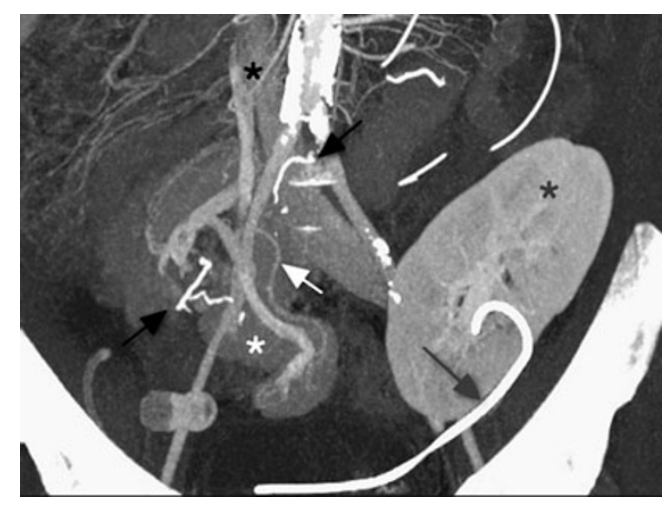

Fig. 3 Coronal multiplanar reformat of SPK transplant, in the portal venous phase of enhancement. Duodenal staple line (black arrows), ureteric stent (grey arrow), renal (grey asterisk) and pancreatic transplant (white asterisk), donor portal vein anastomosed to IVC (black asterisk), splenic artery (white arrow) 


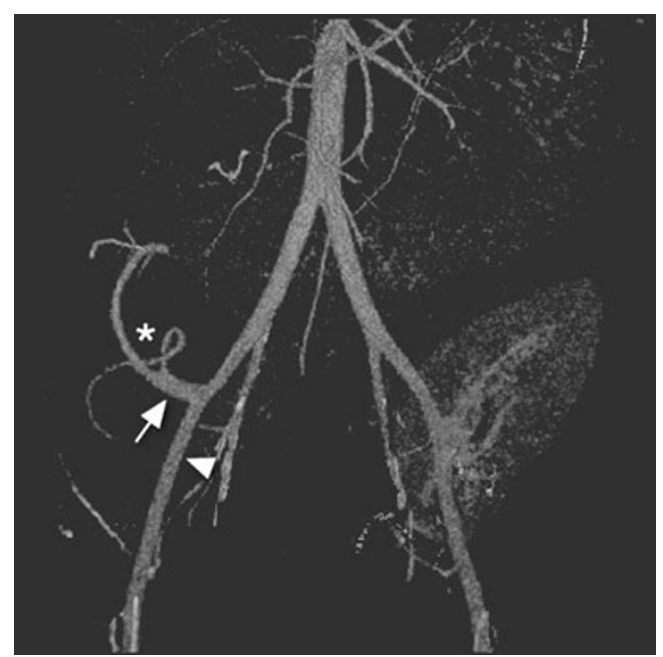

Fig. $4 \mathrm{CT}$ arteriogram demonstrating the 'Y graft' (white asterisk) of the donor splenic artery and SMA anastomosed to donor iliac artery bifurcation (white arrow), which is in turn anastomosed to the recipient external iliac artery (white arrowhead)

depending upon the clinical indication. For instance, either modality could be used if a pseudocyst or collection is suspected.

\section{Complications}

Rejection is a common cause of pancreatic graft failure [17]. For the period 2000-2004, the IPTR reported acute rejection to be the cause of graft failure in $7-25 \%$ of cases. Chronic rejection was reported to be the cause of graft failure in $2-33 \%$ of cases [18]. Rejection has no specific imaging features. The cause of early graft failure is often difficult to determine clinically. Imaging therefore has a vital role in the exclusion of other causes of pancreatic graft failure and in guiding biopsy (Fig. 5).

The potential complications of pancreas transplant may be divided into three categories: those related to the vessels,

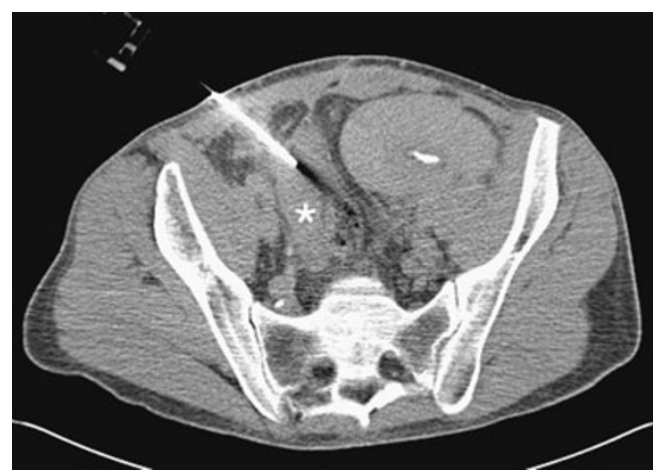

Fig. 5 CT-guided biopsy of pancreatic transplant (white asterisk). Renal transplant seen in left iliac fossa those related to the graft parenchyma and those related to the enteric anastomoses.

\section{Pancreas graft vascular complications}

Graft thrombosis is the most common non-immunological cause of early graft failure. The incidence of graft thrombosis has been reported to range from 2 to $19 \%$ [19-26]. Venous thrombosis is more common than arterial thrombosis [27-29].

In venous thrombosis, US Doppler imaging demonstrates absent venous flow, and a high resistance arterial waveform, with reversed diastolic flow, may also be seen occasionally. Unenhanced CT may show thrombus as a high attenuation portal vein or splenic vein (Fig. 6a). A filling defect may be seen following the adminstration of intravenous contrast medium (Fig. 6b); but subtle thromboses may be obscured following parenchymal enhancement. Side-branch venous thromboses may be seen where small venous side branches have been ligated during retrieval. These are commonly of no clinical significance but are monitored to exclude the presence of thrombus propagation. Venous-graft thrombosis may result in pancreatic necrosis or duodenal stump breakdown, which usually necessitates graft pancreatectomy. Prompt diagnosis and immediate thrombectomy may be successful.

Graft arterial thrombosis is much less common than venous thrombosis, and can occur in the early or late phase post-transplantation. The thrombosis may also be seen as a hyperdense vessel on unenhanced CT, with subsequent non-enhancement following intravenous contrast medium (Fig. 7). Early arterial thrombosis may occur spontaneously at any of the anastomotic sites, or as a consequence of vascular rejection, and results in non-enhancement and subsequent necrosis of the pancreatic graft. Late arterial occlusion may represent the end point of graft rejection. This initially involves the smaller vessels and progresses to involve the larger ones [30].

Emphysematous transformation may occur within a necrotic graft. This is demonstrated on CT as gas locules within the pancreatic graft [31]. It is impossible to differentiate between emphysematous transformation and gas-forming infection on CT. Scintigraphy with radioisotope-labelled white blood cells may be used as a suitable non invasive alternative, where there is clinical uncertainty regarding the presence of graft associated infection (Fig. 8). However, in this situation, surgical graft exploration is usually indicated.

Haemorrhage may result from failure of the arterial anastomoses, but more commonly occurs where the superior mesenteric vessels or splenic vessels have been ligated. A haematoma may be demonstrated as a high attenuation collection on an initial unenhanced CT series. 
Fig. 6 a Unenhanced coronal CT reformat demonstrating high attenuation thrombus within the donor splenic vein (white arrow). $\mathbf{b} \mathrm{CT}$ in the portal venous phase reveals a thrombus within the donor splenic vein (white arrow)
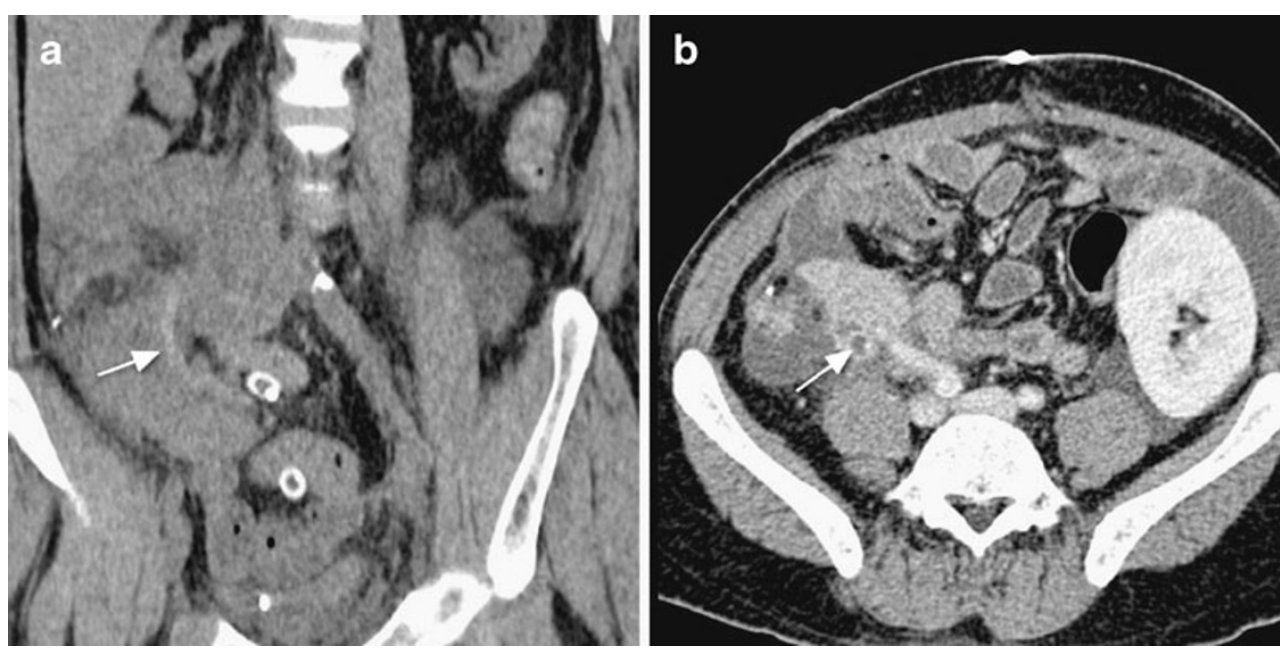

Contrast extravasation may then be detected in the arterial and/or venous phases. While significant intra-abdominal bleeding after pancreas transplantation remains one of the most common reasons for relaparotomy, less than $0.3 \%$ of all pancreas grafts are lost as a result of haemorrhage [32].

Pseudoaneurysms, arteriovenous fistulae, arterial dissection and arterial stenosis represent some rarer vascular complications. Pseudoaneurysms may be the result of surgical technique (particularly if the mesenteric pedicle has been divided with a linear stapling device), infection, severe pancreatitis, or allograft biopsy. Arteriovenous fistulae may be the result of vascular injury, either at surgery or post-biopsy; they are common in the stapled edge of the mesentery where the superior mesenteric

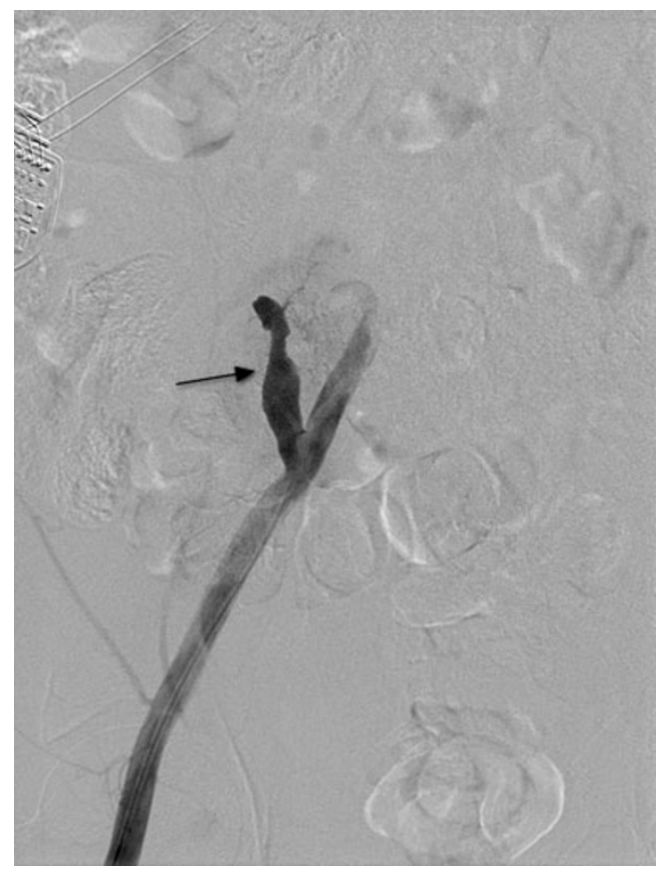

Fig. 7 Conventional angiogram confirming the presence of arterial thrombosis within the pancreatic graft. Only a short segment of the donor common iliac artery (black arrow) is patent vessels have been divided. Arterial stenosis can arise at any anastomosis and may ultimately lead to reduced graft perfusion (Fig. 9). Occasionally, the arterial "Y graft" conduit may kink; this can result in significant haemodynamic compromise and graft dysfunction. Furthermore, the donor portal vein may appear kinked proximal to the anastomosis with the recipient IVC. It is yet to be determined whether this is clinically significant. Our experience suggests that this does not cause graft dysfunction.

CT angiography is the best tool in the initial assessment of these vascular complications. Conventional angiography is used to confirm vascular abnormalities which may then be amenable to endovascular therapy.

\section{Parenchymal complications}

Pancreatic parenchymal graft complications are an important cause of morbidity in the early post-operative period. These complications include acute rejection,

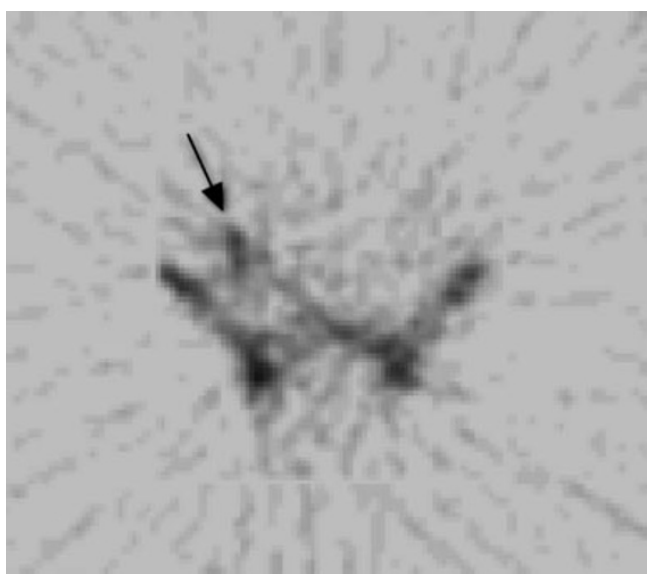

Fig. $8{ }^{111}$ Indium-labelled white cell scintigraphy. Uptake of radioisotope is seen in the right iliac fossa (black arrow), within the infected pancreatic graft 
Fig. 9 a Conventional angiogram reveals a stenosis (black arrow) within the 'Y graft', near anastomoses with the donor common iliac artery. b CT angiogram demonstrates stenosis within the arterial supply (white arrow)
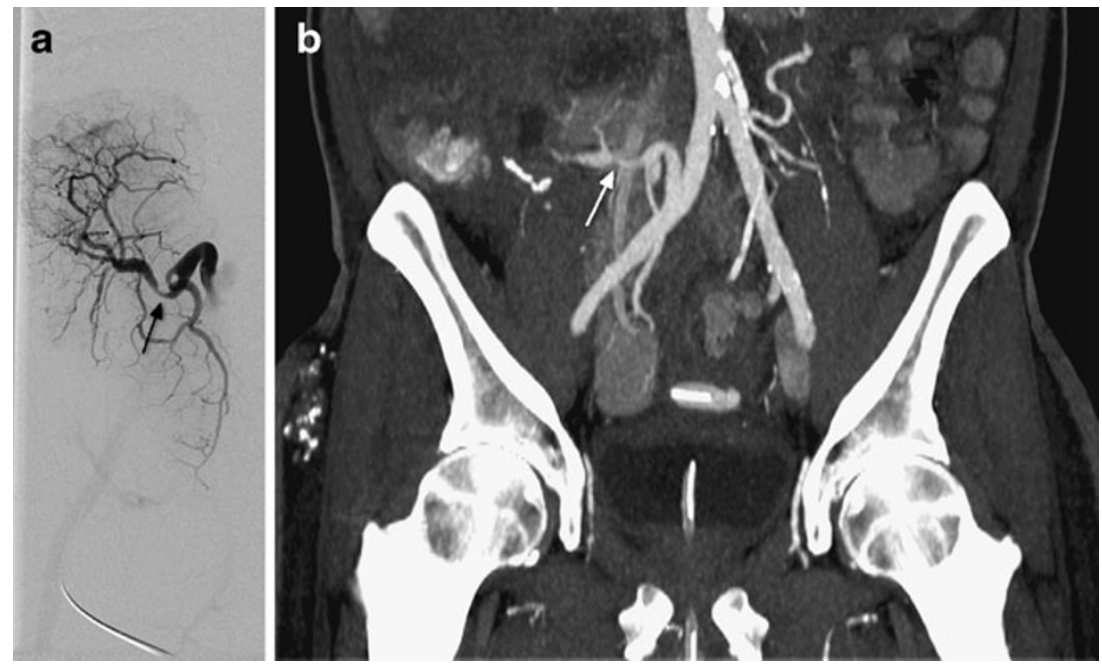

pancreatitis, pseudocyst formation, and infection with abscess formation.

The exact incidence of post-transplant pancreatitis is very difficult to determine because of the lack of a universally accepted definition [33-35]. Prolonged hyperamylasaemia is seen early post transplant in up to $35 \%$ of all recipients [33]. Unfortunately amylase and lipase levels correlate poorly with the severity of graft pancreatitis [33], making evaluation of the true incidence of graft pancreatitis near impossible.

Early graft pancreatitis is often due to reperfusion injury and usually involves the whole graft (Fig. 10). In the early post-operative period, ill-defined fat planes around the pancreatic graft are commonly seen. The graft may appear as an enhancing 'mass' surrounded by an omental wrap in the right iliac fossa. At an early stage it may be difficult to distinguish radiologically between the appearance of the normal post operative pancreatic graft and early graft pancreatitis. Furthermore, focal oedema of the donor's mesenteric fat attached to the SMA stump, presumably the result of donor lymphatic vessel ligation, should not be

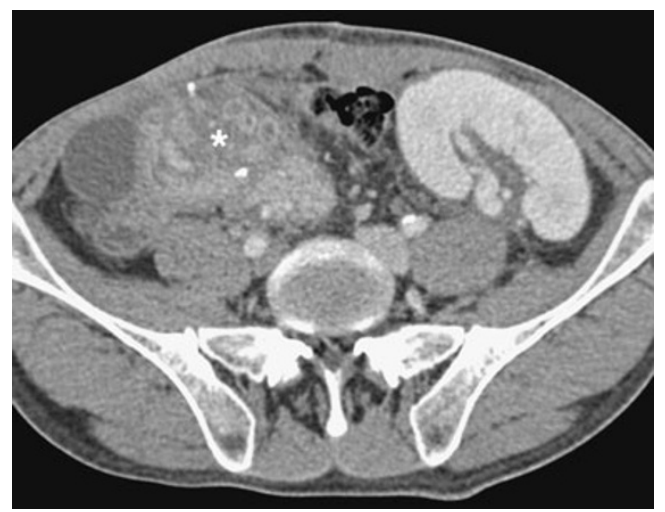

Fig. 10 Axial CT image demonstrating a swollen pancreatic graft (white asterisk), with marked peri-pancreatic fat stranding. Appearances are consistent with graft pancreatitis misinterpreted as focal oedematous pancreatitis [36]. MDCT is valuable in evaluating the complications of pancreatitis, such as parenchymal necrosis and abscess formation. Collections may be amenable to image-guided drainage to alleviate the pressure effect on the vascular supply and drainage of the pancreatic graft.

Repeated episodes of pancreatitis may be secondary to ampullary or duodenal anastomotic stricture or mucus plugging. MRCP may be of diagnostic use in this situation. Mucus plugging may cause focal or generalised duct dilatation, whereas an ampullary or anastomotic stricture will result in generalised duct dilatation in the absence of a pancreatic duct stricture. US can show a bulky pancreas, possibly with associated fluid collections. However, interpretation may be hampered by poorly defined pancreatic margins. Both CT and US may be used to guide percutaneous abscess drainage.

Pseudocysts form at a later stage as a result of graft pancreatitis. They may occur inside or outside the graft and have the potential to become infected. This may result in

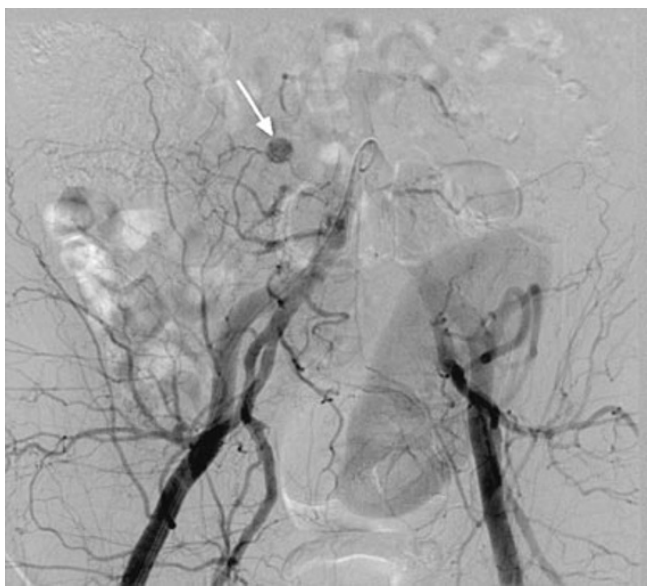

Fig. 11 Conventional angiogram shows a small blush near the head of the pancreatic graft, representing a pseudoaneurysm (white arrow) 


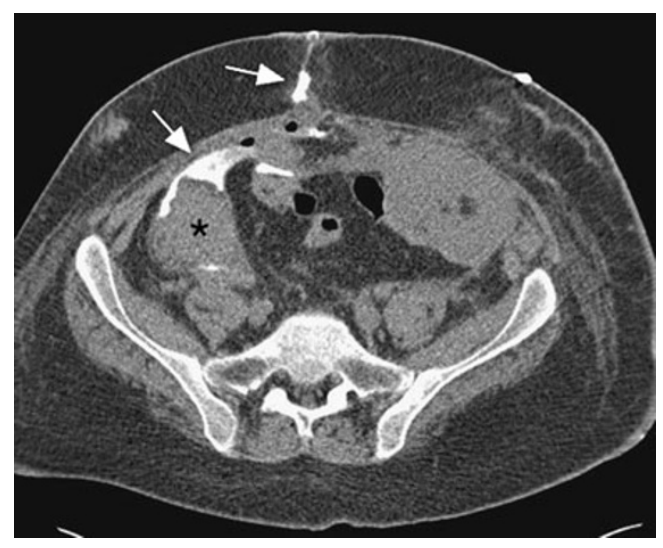

Fig. 12 CT fistulogram reveals an entero-cutaneous fistula postpancreatitis. Contrast medium instilled via the cutaneous fistula demarcates the fistulous tract (white arrows) to the pancreatic head (black asterisk)

vascular pseudoaneurysm formation. The diagnosis should be considered in patients with a pulsatile abdominal mass, unexplained anaemia and/or haemodynamic instability, and when there is rapid enlargement of a pseudocyst. CT angiography is used to evaluate this possibility. Formal angiography is subsequently used to confirm the presence of a pseudoaneurysm (Fig. 11) and permit embolisation.

Another potential complication of pancreatitis is fistula formation. Fistulae may form between the pancreatic graft and the skin or the peritoneal cavity (Fig. 12). Sinus tracts may also develop. Fistulae connecting to the skin are best assessed with CT fistulograms. Internal fistulae are best assessed with MRI.

\section{Enteric complications}

Thickening of the bowel loops surrounding the pancreas graft frequently occurs in the post-operative period, often due to manipulation during the surgical procedure, the

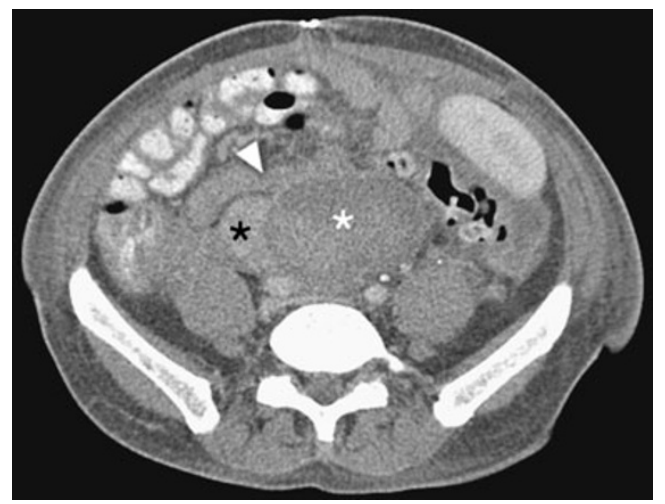

Fig. 13 Axial CT demonstrates a large collection (white asterisk) posterior to the head of the pancreas graft (black asterisk) and donor duodenal loop (white arrowhead). Surgical exploration confirmed a leak at the donor duodenal staple line

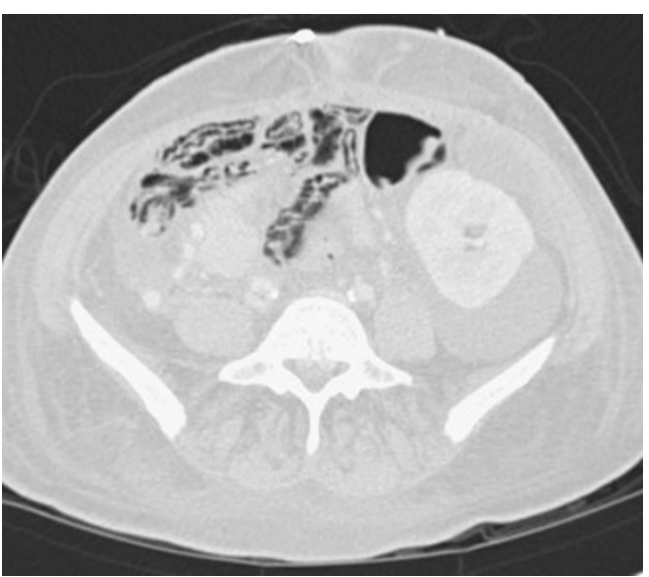

Fig. 14 Axial CT image on "lung windows" reveals extensive pneumatosis coli, secondary to small bowel obstruction at the level of the entero-enterostomy

formation of a Roux loop and aggressive rehydration. This usually resolves within 3-4 weeks. The donor duodenum may also appear thick-walled [37].

Duodenal segment leaks may occur at the duodenojejunostomy, but are more common at the staple line of the duodenal stump (Fig. 13). These occur early due to ischaemia, or late ( $>4$ weeks) secondary to infection or rejection. Free air around the graft is common in the early post-operative period, but seen late may indicate an anastomotic leak. Free fluid may also be seen and collections may form adjacent to the leaking point.

Small bowel obstruction is most commonly secondary to adhesions, a generic risk of abdominal surgery (Fig. 14). Another potential cause is internal herniation of jejunal loops posterior to the pancreatic graft, through a mesenteric defect related to the Roux loop formation [37].

Complications of immunosuppressive therapy may manifest in the bowel. Rarely, a neutropenic typhlitis may develop. Alternatively, opportunistic infections with organ-

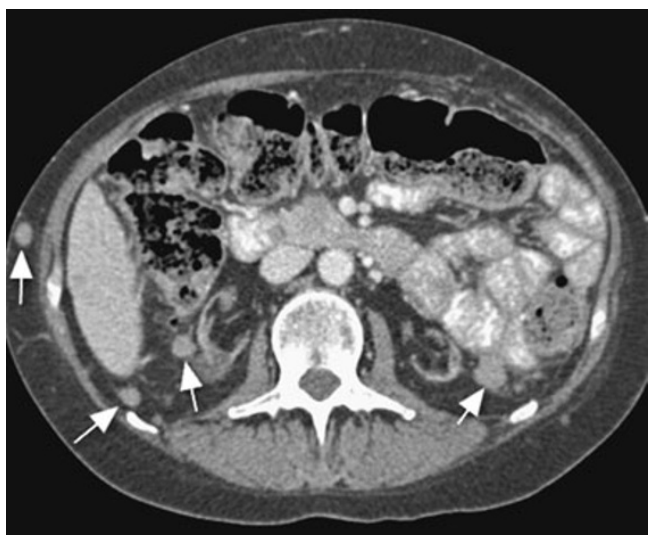

Fig. 15 Axial CT image at the level of the atrophic native kidneys demonstrates multiple soft tissue nodules (white arrows) within the retroperitoneum and subcutaneous tissues. Biopsy confirmed posttransplantation lymphoproliferative disorder 
isms such as cytomegalovirus, or antibiotic-associated Clostridium difficile may cause colitis.

\section{Complications secondary to immunosuppression}

The common complications secondary to immunosuppression seen in solid organ transplantation are generally categorised as related to either infection or tumour. We would like to highlight the specific complication of posttransplant lymphoproliferative disorder (PTLD) that can be diagnosed using cross-sectional imaging. PTLD is a serious but rare complication of pancreas transplantation. It has a reported incidence after pancreatic transplantation of 3-12\% $[38,39]$. It is associated with the higher levels of immunosuppression needed in SPK transplants compared with other solid organ transplants. It is associated with a donor acquired $\mathrm{EBV}$ infection and is therefore commoner in younger previously EBV-naive transplant recipients, for example, type I DM recipients of SPK grafts. Diffuse enlargement of the pancreatic graft is a common manifestation, which is indistinguishable from oedematous pancreatitis or transplant rejection. Less commonly focal intra- or extra-allograft masses may develop (Fig. 15), and lymphadenopathy and other organomegaly may also occur [40]. A tissue diagnosis is essential as the treatment modalities include a reduction in immunosuppression and consideration of chemotherapy.

\section{Conclusion}

With the increasing number of pancreatic transplants that are now being performed, it is vital that radiologists understand the complex post-operative anatomy and the potential complications of transplantation. By doing this, they can use the diagnostic tools available to them to their full potential. It is crucial that surgical and radiology teams liaise in these patients, in order that the radiologists are familiar with the local operative technique. This enables the imaging to be tailored to the patient and will increase the accuracy of image interpretation. In addition, by performing percutaneous drainage procedures, biopsies and endovascular therapies, radiologists have an important role to play in the management of many complications.

\section{References}

1. Gruessner AC, Sutherland DE (2005) Pancreas transplant outcomes for United States (US) and non-US cases as reported to the United Network for Organ Sharing (UNOS) and the International Transplant Registry (IPTR) as of June 2004. Clin Transplant 19:433-455

2. Nath DS, Gruessner AC, Kandaswamy R, Gruessner RW, Sutherland DE, Humar A (2005) Outcomes of pancreas trans- plants for patients with type 2 diabetes mellitus. Clin Transplant 19:792-797

3. Kelly WD, Lillehei RC, Merkel FK, Idezuki Y, Goetz FC (1967) Allotransplantation of the pancreas and duodenum along with the kidney in diabetic nephropathy. Surgery 61:827-837

4. Ojo AO, Meier-Kriesche HU, Hanson JA, Leichtman A, Magee JC, Cibrik D et al (2001) The impact of simultaneous pancreaskidney transplantation on long-term patient survival. Transplantation 71:82-90

5. Farney AC, Cho E, Schweitzer EJ, Dunkin B, Philosophe B, Colonna J et al (2000) Simultaneous cadaver pancreas livingdonor kidney transplantation: a new approach for type 1 diabetic uremic patient. Ann Surg 232:696-03

6. Gruessner RW, Sutherland DE (1996) Simultaneous kidney and segmental pancreas transplants from living related donors-the first two successful cases. Transplantation 61:1265-1268

7. Sutherland DE, Gruessner RW, Dunn DL, Matas AJ, Humar A, Kandaswamy R et al (2001) Lessons learnt from more than 1, 000 pancreas transplants at a single institution. Ann Surg 233:463-501

8. Freund MC, Steurer W, Gassner EM, Unsinn KM, Rieger M, Koenigsrainer A et al (2004) Spectrum of imaging findings after pancreas transplantation with enteric exocrine drainage: part 1, posttransplantation anatomy. AJR 182:911-917

9. Zielinski A, Nazarewski S, Bogetti D, Sileri P, Testa G, Sankary H et al (2003) Simultaneous pancreas-kidney transplant from living related donor: a single-center experience. Transplantation 76 (3):547-552

10. Gruessner RW, Kendall DM, Drangstveit MB, Gruessner AC, Sutherland DE (1997) Simultaneous pancreas-kidney transplantation from live donors. Ann Surg 226(4):471-482

11. Nikolaidis P, Amin RS, Hwang CM, McCarthy RM, Clark JH, Gruber SA et al (2003) Role of sonography in pancreatic transplantation. Radiographics 23:939-949

12. Aideyan OA, Foshager MC, Benedetti E, Troppmann C, Gruessner RW (1997) Correlation of the arterial resistive index in pancreas transplants of patients with transplant rejection. AJR Am J Roentgenol 168:1445-1447

13. Wong JJ, Krebs TL, Klassen DK, Daly B, Simon EM, Bartlett ST et al (1996) Sonographic evaluation of acute pancreas transplant rejection: morphology-Doppler analysis versus guided percutaneous biopsy. AJR Am J Roentgenol 166:803-807

14. Dachmann AH, Newmark GM, Jnr TJR et al (1998) Imaging of pancreatic transplantation using portal venous and enteric exocrine drainage. AJR Am J Roentgenol 171:157-163

15. Gupta R, Rottenburg G, Taylor J (2002) Pseudothrombosis of the iliac vein in patients following combined kidney and pancreas transplantation. Br J Radiol 75:692-694

16. Heverhagen JT, Wagner HJ, Ebel H, Levine AL, Klose KJ, Hellinger A (2004) Pancreatic transplants: noninvasive evaluation with secretin-augmented $\mathrm{mr}$ pancreatography and MR perfusion measurements - preliminary results. Radiology 233 (1):273-280

17. Waki K, Kadowaki T (2007). An analysis of long-term survival from the OPTN/UNOS Pancreas Transplant Registry. Clin Transplant 9-17

18. International Pancreas Transplant Registry (IPTR). (2004) Annual Report. Available via http://www.iptr.umn.edu/home.html

19. Sollinger HW, Knechtle SJ, Reed A, D'Alessandro AM, Kalayoglu M, Belzer FO et al (1991) Experience with 100 consecutive simultaneous kidneypancreas transplants with bladder drainage. Ann Surg 214:703-711

20. Tollemar J, Tyden G, Brattstrom C, Groth CG (1988) Anticoagulation therapy for prevention of pancreatic graft thrombosis: benefits and risks. Transplant Proc 20:479-480

21. Douzdjian V, Abecassis MM, Cooper JL, Argibay PF, Smith JL, Corry RJ (1993) Pancreas transplant salvage after acute venous thrombosis. Transplantation 56(1):222-223 
22. Troppmann C, Gruessner AC, Benedetti E, Papalois BE, Dunn DL, Najarian JS et al (1996) Vascular graft thrombosis after pancreatic transplantation: univariate and multivariate operative and nonoperative risk factor analysis. J Am Coll Surg 182(4):285316

23. Gilabert R, Fernández-Cruz L, Real MI, Ricart MJ, Astudillo E, Montaña X (2002) Treatment and outcome of pancreatic venous graft thrombosis after kidney-pancreas transplantation. Br J Surg $89: 355-360$

24. Decker E, Coimbra C, Weekers L, Detry O, Honoré P, Squifflet JP et al (2009) A retrospective monocenter review of simultaneous pancreas-kidney transplantation. Transplant Proc 41(8):33893392

25. Gruessner RW, Sutherland DE, Troppmann C, Benedetti E, Hakim N, Dunn DL et al (1997) The surgical risk of pancreas transplantation in the cyclosporine era: an overview. J Am Coll Surg 185(2):128-144

26. Sansalone CV, Maione G, Aseni P, Mangoni I, De Roberto A, Soldano S et al (2005) Surgical complications are the main cause of pancreatic allograft loss in pancreas-kidney transplant recipients. Transplant Proc 37:2651-2653

27. Chandra J, Phillips RR, Boardman P, Gleeson FV, Anderson EM (2009) Pancreas transplants. Clin Radiol 64(7):714-723

28. Nicoluzzi J, Silveira F, Porto F, Macri M (2009) One hundred pancreas transplants performed in a Brazilian institution. Transplant Proc 41:4270-4273

29. Eubank WB, Schmiedl UP, Levy AE, Marsh CL (2000) Venous thrombosis and occlusion after pancreas transplantation: evaluation with breath-hold gadolinium-enhanced three-dimensional MR imaging. AJR Am J Roentgenol 175(2):381-385

30. Krebs TL, Daly B, Wong-You-Cheong JJ, Carroll K, Bartlett ST (1999) Acute pancreatic transplant rejection: evaluation with dynamic contrast-enhanced MR imaging compared to histopathologic analysis. Radiology 210:437-442

31. Vas W, Patel B, Mahanta B, Salimi Z, Markivee C, Garvin P (1989) Innocuous gas collections in pancreatic allografts demonstrated by computed tomography. Gastrointest Radiol 14:118-122
32. Gruessner AC, Sutherland DER (2009) Pancreas transplant outcomes for United States (US) cases as reported to the United Network for Organ Sharing (UNOS) and the International Pancreas Transplant Registry (IPTR). In: Cecka JM, Terasaki (eds) Clinical transplants 2008. UCLA Tissue Typing Laboratory, Los Angeles, pp 45-56

33. Troppmann C (2004) Surgical complications. In: Gruessner RWG, Sutherland DER (eds) Pancreas transplantation. Springer, New York, pp 206-237

34. Troppmann C, Gruessner AC, Dunn DL, Sutherland DE, Gruessner RW (1998) Surgical complications requiring early relaparotomy after pancreas transplantation: a multivariate risk factor and economic impact analysis of the cyclosporine era. Ann Surg 227:255-268

35. Small RM, Shetzigovski I, Blachar A, Sosna J, Klausner JM, Nakache R et al (2008) Redefining late acute graft pancreatitis: clinical presentation, radiologic findings, principles of management, and prognosis. Ann Surg 247:1058-1063

36. Freund MC, Steurer W, Gassner EM, Unsinn KM, Rieger M, Koenigsrainer A et al (2004) Spectrum of imaging findings after pancreas transplantation with enteric exocrine drainage: part 2, Posttransplantation complications. AJR Am J Roentgenol 182:919-925

37. Lall CG, Sandrasegaran K, Maglinte DT, Fridell JA (2006) Bowel complications seen on CT after pancreas transplantation with enteric drainage. AJR Am J Roentgenol 187:1288-1295

38. Lumbreras C, Fernandez I, Velosa J, Munn S, Sterioff S, Paya CV (1995) Infectious complications following pancreatic transplantation: incidence, microbiological and clinical characteristics, and outcome. Clin Infect Dis 20:514-520

39. Martinenghi S, Dell'Antonio G, Secchi A, Di Carlo V, Pozza G (1997) Cancer arising after pancreas and/or kidney transplantation in a series of 99 diabetic patients. Diab Care 20:272-275

40. Meador TL, Krebs TL, Cheong JJ, Daly B, Keay S, Bartlett S (2000) Imaging features of posttransplantation lymphoproliferative disorder in pancreas transplant recipients. AJR Am J Roentgenol 174:121-124 